\title{
A Survey on Reversible Image Data Hiding with Quality Enhancement
}

\author{
Nishi Vishwakarma \\ MTech Scholar, Department of \\ Electronics \& Communication \\ Engineering, Truba Institute of \\ Engineering and Information \\ Technology Bhopal
}

\author{
Neelesh Gupta \\ H.O.D, Department of \\ Electronics \& Communication \\ Engineering, Truba Institute of \\ Engineering and Information \\ Technology Bhopal
}

\author{
Neetu Sharma \\ Asst. Prof., Department of \\ Electronics \& Communication \\ Engineering, Truba Institute of \\ Engineering and Information \\ Technology Bhopal.
}

\begin{abstract}
In this paper, a completely unique reversible data hiding $(\mathrm{RDH})$ algorithmic rule is projected for digital images. rather than attempting to keep the PSNR value high, the projected algorithmic rule enhances the contrast of a host image to boost its visual quality. the best 2 bins within the histogram are selected for information embedding in classify that histogram equalisation determination be execute by repeating the method. The side info is embedded in conjunction with the message bits into the host image so the initial image is totally retrievable. The projected algorithmic rule was implemented on 2 sets of images to demonstrate its efficiency. To our greatest knowledge, it's the primary algorithmic rule that achieves image contrast enhancement byRDH. moreover, the analysis results show that the visual quality is preserved once a considerable amount of message bits have been embedded into the contrast-enhanced images, even higher than 3 specificMATLAB functions used for image contrast enhancement.
\end{abstract}

\section{Keywords}

Contrast enhancement, histogram modification, location map, reversible data hiding, and visual quality.

\section{INTRODUCTION}

Reversible data hiding (RDH) has been intensively studied within the community of signal process. additionally referred as invertible or lossless information hiding, $\mathrm{RDH}$ is to embed a piece of data into a host signal to get the marked one, from that the first signal will be exactly recovered after extracting the embedded data. The technique of RDH is helpful in some sensitive applications wherever no permanent modification is allowed on the host signal. within the literature, most of the projected algorithms are for digital pictures to embed invisible information (e.g. [1]-[8]) or a visible watermark (e.g. [9]). to judge the performance of a RDH algorithmic rule, the hiding rate and therefore the marked image quality are necessary metrics. There exists a trade-off between them as a result of increasing the hiding rate usually causes more distortion in image content. to measure the distortion, the peak signal/noise ratio (PSNR) worth of themarked image is usually calculated. usually speaking, direct modification of image histogram [2] provides less embedding capacity. In contrast, the more moderen algorithms (e.g. [5]-[8]) manipulate the additional centrally distributed prediction errors by exploiting the correlations between neighboring pixels so less distortion is caused by information hiding. though the PSNR of a marked image generated with a prediction error based mostly algorithmic rule is kept high, the visual quality will hardly be improved because more or less distortion has been introduced by the embedding operations. For the pictures acquired with poor illumination, raising the visual quality is more necessary than keeping the PSNR value high. Moreover, contrast improvement of medical or satellite pictures is desired to point out the main points for visual examination. though the PSNR value of the enhanced image is usually low, the visibility of image details has been improved. To our greatest information, there's no existing RDH algorithmic rule that performs the task of contrast enhancement thus on improve the visual quality of host pictures. thus during this study, we tend to aim at inventing a replacement RDH algorithmic rule to realize the property of contrast enhancement rather than simply keeping the PSNR value high. in principle, image contrast enhancement will be achieved by histogram equalisation [10]. To perform information embedding and contrast enhancement at a similar time, the projected algorithmic rule is performed bymodifying the histogram of pixel values. Firstly, the 2 peaks (i.e. the best 2 bins) within the histogram are detected. The bins between the peaks are unchanged whereas the outer bins are shifted outward so every of $\{$ the two|the 2$\}$ peaks may be split into two adjacent bins. to extend the embedding capability, the very best 2 bins in themodified histogram are often more chosen to be split, then on until satisfactory contrast enhancement effect is achieved. To avoid the overflows and underflows because of histogram modification, the bounding pixel values are preprocessed and a location map is generated to memorise their locations. For the recovery of the original image, the situation map is embedded into the host image, together with|in conjunction with|beside|at the side of|along with\} the message bits and different side information. therefore blind knowledge extraction and complete recovery of the original image are each enabled. The planned algorithmic rule was applied to 2 set of pictures to demonstrate its efficiency. To our best information, it's the first algorithmic rule that achieves image contrast enhancement by RDH. furthermore, the evaluation results show that the visual quality will be preserved after a considerable quantity of message bits are embedded into the contrast-enhanced pictures, even higher than 3 specific MATLAB functions used for image contrast enhancement.

\section{LITERATURE SURVEY}

Hao-Tian Wu et. al. [1] "Reversible Image Data Hiding with Contrast Enhancement” A novel reversible data hiding (RDH) algorithmic rule is projected for digital images. rather than attempting to stay the PSNR value high, the projected algorithmic rule enhances the contrast of a host image to boost its visual quality. the highest 2 bins within the histogram are selected for information embedding so that histogram equalisation will be performed by repetition the method. The side info is embedded along with the message bits into the host image so the original image is totally recoverable. The 
projected algorithmic rule was implemented on 2 sets of pictures to demonstrate its efficiency. To our best data, it's the primary algorithmic rule that achieves image contrast enhancement by RDH. a brand new reversible information hiding algorithmic rule has been proposed with the property of contrast enhancement. Basically, the 2 peaks (i.e. the highest 2 bins) within the histogram are selected for data embedding so histogram equalisation may be at the same time performed by repetition the method. The experimental results have shown that the image contrast will be enhanced by splitting a number of histogram peaks pair by pair. Compared with the special MATLAB functions, the visual quality of the contrast-enhanced pictures generated by our algorithmic rule is better preserved. Moreover, the original image will be exactly recovered without any additional info.

Xinpeng Zhang et. al. [2] "Reversible Data Hiding in Encrypted Image" This work proposes a novel reversible data hiding scheme for encrypted image. After encrypting the entire data of an uncompressed image by a stream cipher, the additional data can be embedded into the image by modifying a small proportion of encrypted data.With an encrypted image containing additional data, one may firstly decrypt it using the encryption key, and the decrypted version is similar to the original image. According to the data-hiding key, with the aid of spatial correlation in natural image, the embedded data can be successfully extracted and the original image can be perfectly recovered. In this work, a novel reversible data hiding scheme for encrypted image with a low computation complexity is proposed, which consists of image encryption, data embedding and dataextraction/image-recovery phases. The data of original image are entirely encrypted by a stream cipher. Although a data-hider does not know the original content, he can embed additional data into the encrypted image by modifying a part of encrypted data. With an encrypted image containing embedded data, a receiver may firstly decrypt it using the encryption key, and the decrypted version is similar to the original image. According to the datahiding key, with the aid of spatial correlation in natural image, the embedded data can be correctly extracted while the original image can be perfectly recovered.

Wei-Liang Tai et. al. [3] "Reversible Data Hiding Based on Histogram Modification of Pixel Differences" In this paper, we tend to present a reversible information hiding scheme based on histogram modification. we have a tendency to exploit a binary tree structure to resolve the problem of communication pairs of peak points. Distribution of pixel differences is employed to realize large hiding capacity whereas keeping the distortion low. we also adopt a histogram shifting technique to stop overflow and underflow. Performance comparisons with different existing schemes are provided to demonstrate the prevalence of the proposed scheme. during this letter, we've presented an efficient extension of the histogram modification technique by considering the differences between adjacent pixels instead of easy pixel value. One common disadvantage of virtually all histogram modification techniques is that they need to provide a side channel for pairs of peak and minimum points. to resolve this problem, we have a tendency to introduced a binary tree that predetermines the multiple peak points used to embed messages; thus, the only info the sender and recipient must share is that the tree level $\mathrm{L}$.

Jun Tian et. al. [4] "Reversible Data Embedding Using a Difference Expansion" Reversible data embedding has drawn a lot of interest recently. Being reversible, the original digital content may be utterly restored . during this paper, we have a tendency to present a completely unique reversible dataembedding technique for digital images. We explore the redundancy in digital pictures to realize very high embedding capacity, and keep the distortion low. in this paper, we've got presented a simple and efficient reversible date-embedding technique for digital images. we have a tendency to explored the redundancy within the digital content to realize reversibility. both the payload capability limit and therefore the visual quality of embedded images are among the simplest within the literature.

Yongjian $\mathrm{Hu}$ et. al. [5] "DE-Based Reversible Data Hiding with Improved Overflow Location Map" For differenceexpansion (DE)-based reversible data hiding, the embedded bit-stream mainly consists of two parts: one part that conveys the secret message and the other part that contains embedding information, including the 2-D binary (Overflow) location map and the header file. The first part is the payload while the second part is the auxiliary information package for blind detection. To increase embedding capacity, we have to make the size of the second part as small as possible. Classical DE method has a large auxiliary information package. The problem by using a payload-independent overflow location map. However, the compressibility of the overflow location map is still undesirable in some image types. In this paper, we focus on improving the overflow location map. We design a new embedding scheme that helps us construct an efficient payload-dependent overflow location map. Such an overflow location map has good compressibility. Our accurate capacity control capability also reduces unnecessary alteration to the image. In this paper, we have investigated the problem of the overflow location map, which is an important issue in DEbased reversible data hiding. In order to increase embedding capacity, we improve the compressibility of the overflow location map by designing a new embedding scheme. Our overflow location map depends on the payload. Unlike other (overflow) location maps, it contains two types of overflowlocations: one from embedding and the other from shifting. Since the constraint on shifting is looser than that on embedding, it results in fewer overflow locations. Therefore, our overflow location map matrix is often sparser than other current ones. This feature makes our overflow location map have higher compressibility.

\section{METHOD}

\section{$\mathrm{RDH}$ algorithmic rule with contrast enhancement}

\section{A. Data Embedding by Histogram Modification}

The algorithmic rule to be presented is primarily for graylevel images however will be easily extended to color images. Given an 8-bit gray-level image, the image histogram will be calculated by count the pixels with a gray-level value $\mathrm{j}$ For $\mathrm{j}$. we have a tendency to use hI to denote the image histrogram so $h I$ (j) represent the number of pixels with a value $j$. Suppose I contains N totally different pixel values. Then there are $\mathrm{N}$ nonempty bins in hI, from which the 2 peak (i.e. the highest 2 bins) are chosen and therefore the corresponding smaller and bigger values are denoted by IS and IR, respectively. For a pixel counted in hI with value $\mathrm{i}$, information embedding is performed by 


$$
i^{\prime}= \begin{cases}i-1, & \text { for } i<I_{S} \\ I_{S}-b_{k}, & \text { for } i=I_{S} \\ i, & \text { for } I_{S}<i<I_{R} \\ I_{R}+b_{k}, & \text { for } i=I_{R} \\ i+1, & \text { for } i>I_{R},\end{cases}
$$

Wherever the modified pixel is value, and is the k-th message bit ( 0 or 1$)$ to be hidden. By applying eq. (1) to each pixel counted in hI, completely hI (IS) + hI (IR) binary values are embedded. Provided that there's no bounding value (0 or 255) in I (otherwise pre-process is needed), there'll be bins within the modified histogram. That is, the bins between \{ the 2 |the 2 ) peaks are unchanged whereas the outer ones are shifted outward so that every of the peaks are often split into two adjacent bins (i.e. and, and, respectively). the peak values IS and IR has to be provided to extract the embedded information. a technique to stay them is to exclude sixteen pixels in I from histogram computing. the least significant bits (LSB) of these pixels are collected and included within the binary values to be hidden. When applying eq. (1) to each pixel counted in hI for information embedding, the values of IS and IR (each with eight bits) are used to replace the LSBs of the sixteen excluded pixels by bitwise operation. To extract the embedded knowledge, the height values ought to be retrieved and therefore the histogram of the marked image is calculated excluding the sixteen pixels aforesaid. Then the following operation is performed on any pixel counted within the histogram and with the value of $I_{S}-1, I_{S}, I_{R}$ or $I_{R}+1$ :

$$
b_{k}^{\prime}=\left\{\begin{array}{cl}
1, & \text { if } i^{\prime}=I_{S}-1 \\
0, & \text { if } i^{\prime}=I_{S} \\
0, & \text { if } i^{\prime}=I_{R} \\
1, & \text { if } i^{\prime}=I_{R}+1,
\end{array}\right.
$$

Where the k-th binary value is extracted from the marked image I'. The extraction operations are performed within the same order as that of the embedding operations. according to eq. (1), the following operation is performed on every pixel counted within the histogram to recover its original value:

$$
i=\left\{\begin{array}{l}
i^{\prime}+1, \quad \text { for } i^{\prime}<I_{S}-1 \\
I_{S}, \quad \text { for } i^{\prime}=I_{S}-1 \text { or } i^{\prime}=I_{S} \\
I_{R}, \quad \text { for } i^{\prime}=I_{R} \text { or } i^{\prime}=I_{R}+1 \\
i^{\prime}-1, \quad \text { for } i^{\prime}>I_{R}+1,
\end{array}\right.
$$

The location map will be pre-computed and compressed to be first of all embedded into the host image. the value of , the dimensions of the compressed location map, and therefore the previous peak values, in contrary, are embedded with the last 2 peaks to be split,whose values are keep within the LSBs of the sixteen excluded pixels. within the extraction method, the last split peak values are retrieved and therefore the information embedded with them are extracted with eq. (2). when restoring the histogram with eq. (3), the information embedded with the previously split peaks may also be extracted by process them pair by pair. At last, the location map is obtained from the extracted data to identify the pixel values modified within the pre-process.

\section{B. Procedure of the proposed algorithmic rule}

The procedure of the proposed algorithmic rule is illustrated in Fig. 1. provided that completely $\mathrm{L}$ pairs of histogram bins are to be split for data embedding, the embedding procedure includes the following steps:

1) Pre-process: The pixels within the vary of $[0, \mathrm{~L}-1]$ and [256-L, 255] are processed as mentioned in Section II-C excluding the first sixteen pixels within the bottom row. A location map is generated to record the locations of these pixels and compressed by the JBIG2 standard [11] to reduce its length.

2) The image histogram is calculated without count the first sixteen pixels within the bottom row.

3) Embedding: the 2 peaks (i.e. the highest 2 bins) within the histogram are split for data embedding by applying eq. (1) to each pixel counted within the histogram. Then the 2 peaks within the modified histogram are chosen to be split, and then on until pairs are split. The bitstream of the compressed location map is embedded before the message bits (binary values). the value of, the length of the compressed location map, the LSBs collected from the sixteen excluded pixels, and therefore the previous peak values are embedded with the last 2 peaks to be split.

4) The lastly split peak values are used to replace the LSBs of the sixteen excluded pixels to create the marked image.

The extraction and recovery method include the following steps:

A) The LSBs of the sixteen excluded pixels are retrieved so the values of the last 2 split peaks are known .

B) the information embedded with the last 2 split peaks are extracted by using eq. (2) so the value of, the length of the compressed location map, the original LSBs of sixteen excluded pixels, and therefore the previously split peak values are known . Then the recovery operations are carried out by process all pixels except the sixteen excluded ones with eq. (3). the process of extraction and recovery is repeated until all of the split peaks are restored and therefore the data embedded with them are extracted.

C) The compressed location map is obtained from the extracted binary values and decompressed to the original size.

D) With the decompressed map, those pixels changed in preprocess are identified. Among them, a pixel value is subtracted by if it's less than 128 , or increased by otherwise. To comply with this rule, the maximum value of is sixty four to avoid ambiguity. At last, the original image is recovered by writing back the original LSBs of sixteen excluded pixels.

\section{CONCLUSION}

In this paper, a brand new reversible information hiding algorithmic rule has been projected with the property of contrast enhancement. Basically, the two peaks (i.e. the highest 2 bins) within the histogram are selected for information embedding so histogram equalisation are often at the same time performed by repetition the method. 


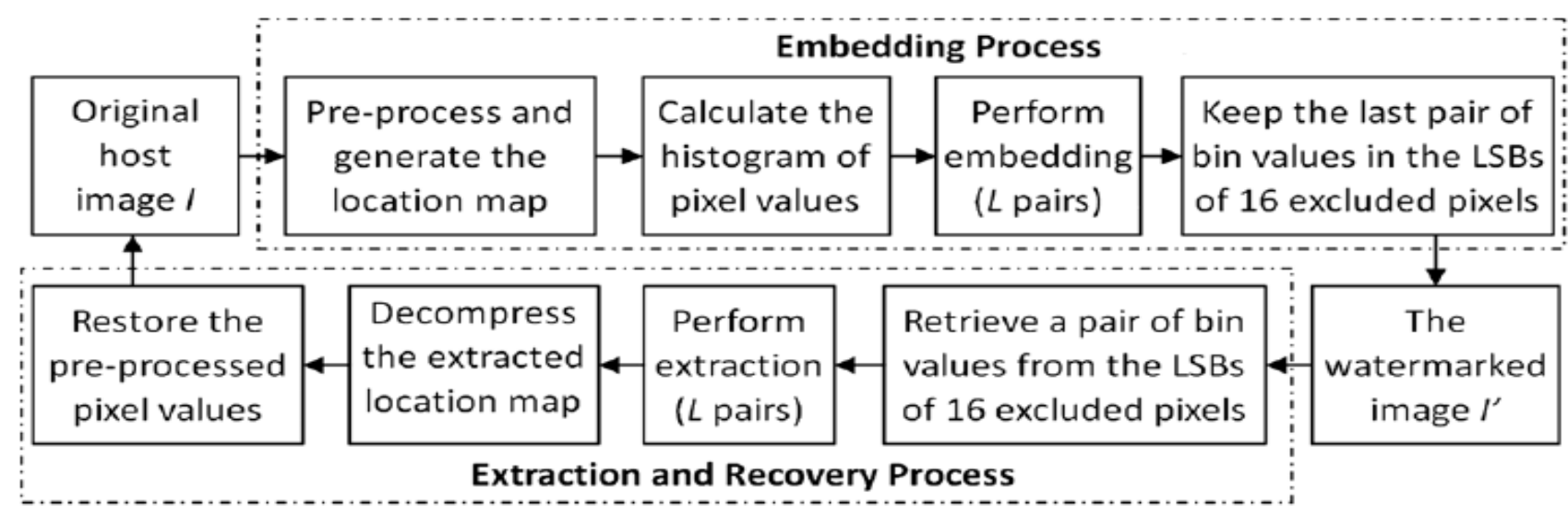

Fig. 1. Procedure of the proposed RDH algorithm

The experimental results have shown that the image contrast are often enhanced by splitting a number of histogram peaks pair by pair. Compared with the special MATLAB functions, the visual quality of the contrast-enhanced images generated by our algorithmic rule is better preserved. Moreover, the original image are often exactly recovered without any additional info. hence the projected algorithmic rule has created the image contrast enhancement reversible. improving the algorithmic rule robustness, and applying it to the medical and satellite images for the better visibility, are going to be our future work.

\section{REFERENCES}

[1] Hao-Tian Wu, Jean-Luc Dugelay, Yun-Qing Shi, "Reversible Image Data Hiding with Contrast Enhancement" IEEE signal processing letters, vol. 22, no. 1 , january 2015

[2] Xinpeng Zhang "Reversible Data Hiding in Encrypted Image" IEEE signal processing letters, vol. 18, no. 4, april 2011

[3] Wei-Liang Tai, Chia-Ming Yeh, Chin-Chen Chang "Reversible Data Hiding Based on Histogram Modification of Pixel Differences" IEEE transactions on circuits and systems for video technology, vol. 19, no. 6, june 2009

[4] Jun Tian "Reversible Data Embedding Using a Difference Expansion" IEEE transactions on circuits and systems for video technology, vol. 13, no. 8, august 2003
[5] Yongjian Hu, Heung-Kyu Lee, Jianwei Li. "DE-Based Reversible Data Hiding with Improved Overflow Location Map" IEEE transactions on circuits and systems for video technology, vol. 19, no. 2, february 2009

[6] Z. Zhao, H. Luo, Z-M. Lu. and J.s.pan, "Reversible data hiding based on multilevel histrogram and sequential recovery" Int. J. Electron. Commun. (AEU), Vol. 65, pp. 814-826, 2011.

[7] M. Kuribayashi and H. Tanaka, "Fingerprinting protocol for images based on additive homomorphic property," IEEE Trans. Image Process., vol. 14, pp. 2129-2139, 2005.

[8] Y.T. N. Memon and P. W. Wong, "A buyer-seller watermarking protocol," IEEE Trans. Image Process., vol. 10, no. 4, pp. 643-649, Apr. 2001

[9] S. Lian, Z. Liu, Z. Ren, and H. Wang, "Commutative encryption and watermarking in video compression," IEEE Trans. Circuits Syst. Video Technol., vol. 17, no. 6, pp. 774-778, 2007

[10] L. Luo, Z. Chen, M. Chen, X. Zeng, and Z. Xiong, "Reversible image watermarking using interpolation technique," IEEE Trans. Inf. Forensics Secur., vol. 5, no. 1, pp. 187-193, 2010. 